\title{
BIOINCRUSTANTES EN ESTRUCTURAS DE CULTIVO DE Argopecten purpuratus EN BAHÍA SAMANCO, PERÚ
}

\section{BIOFOULING IN Argopecten purpuratus CULTURE STRUCTURES AT SAMANCO BAY, PERU}

\author{
Aldo Pacheco ${ }^{1}$ y Antonio Garate ${ }^{2}$
}

\begin{abstract}
Resumen
Los bioincrustantes, son organismos que se adhieren sobre estructuras duras que se sumergen o están en contacto continuo con el agua de mar. Con el objetivo de dar a conocer la composición de especies bioincrustantes en el cultivo suspendido de Argopecten purpuratus, el día 20 de Octubre de 1998 se realizó un muestreo de diferentes materiales y sobre las valvas de las conchas, en la Concesión de Maricultura de la Universidad Ricardo Palma (Bahía Samanco, Ancash, Perú). Se identificaron un total de 33 especies pertenecientes a 10 grupos taxonómicos. Las diferentes especies correspondieron a ambientes, intermareales, submareales y pelágicos.
\end{abstract}

Palabras clave: Bioincrustantes, Argopecten purpuratus, estructuras de cultivo, Bahía Samanco.

\begin{abstract}
Biofouling are species that are attached to hard substrata that are immersed or in permanent contact with the sea water. With the objective of assessing the biofouling species composition in an Argopecten purpuratus suspended culture, organisms were sampled in October 20, 1998 at the Universidad Ricardo Palma sea farm (Samanco Bay, Ancash, Peru). A total of 33 species belonging to 10 taxonomical groups were identified. The species corresponded to intertidal, subtidal and pelagic habitats.
\end{abstract}

Key words: Biofouling, Argopecten purpuratus, culture structures, Samanco Bay

\section{Introducción}

Dado que la pesquería de la "concha de abanico" Argopecten purpuratus ha declinado sus poblaciones, su cultivo ha cobrado una gran importancia en los últimos años (Avendaño et al., 2001). Un factor que incide de manera importante en esta actividad, pero que ha recibido menos atención en cuanto a estudios se refiere, son las especies bioincrustantes. Se denomina así a organismos de diferentes taxas, principalmente invertebrados, que colonizan diferentes estructuras duras artificiales que se mantienen en permanente contacto con el agua (por ejemplo; cascos de barcos, muelles, etc.). Estas especies pueden utilizar como sustrato las estructuras del cultivo suspendido de bivalvos; linternas, pearl nets, boyas, bolsas colectoras y cuerdas, así como también las mismas valvas (Perea et al., 1990; Lesser et al., 1992).

$\mathrm{Al}$ desarrollarse en gran abundancia, estas especies pueden afectar al cultivo de manera negativa ya que desgastan y deterioran los materiales, reduciéndoles el tiempo de duración útil. También afectan la flotabilidad, lo que representa un importante costo por mantenimiento de estos materiales (Claereboudt et al. 1994). Además los bioincrustantes puede cubrir totalmente la superficie del material donde se encuentran alojadas las conchas, lo que modifica el flujo de agua, dejándolas sin el suministro de alimento, pudiendo afectar negativamente el crecimiento (Lesser et al., 1992; Claereboudt et al.,
1994; Uribe et al., 2001). Sin embargo Widman \& Rodhes (1991) afirman que un incremento en la abundancia de los bioincrustantes no es un factor de mortalidad importante en cultivos de Argopecten irradians. Incluso, en áreas en donde las corrientes son fuertes, los bioincrustantes puede favorecer el crecimiento, reduciendo la fuerza de las corrientes y facilitando la alimentación (Ross et al., 2002).

Otros autores como Thouzeau (1991) y Aguilar \& Mendo (2002) analizan los bioincrustantes que asientan en las bolsas colectoras para estudiar las interacciones bióticas, buscando optimizar la captación de postlarvas de Pecten maximus y $A$. purpuratus respectivamente. Los bioincrustantes también se asientan en las mismas valvas, lo que afecta la formación y crecimiento de la misma (Perea et al., 1990).

En general no se ha evidenciado de manera concreta, la forma como los bioincrustantes puede afectar la producción del cultivo de A. purpuratus, siendo importante conocer las especies que lo conforman, para posteriormente identificar los diferentes procesos que pueden mediar. La presente nota es un estudio preliminar que tiene como objetivo dar a conocer las especies y hábitos tróficos de los bioincrustantes, que se desarrollan en el cultivo de $A$. purpuratus en Bahía Samanco. 


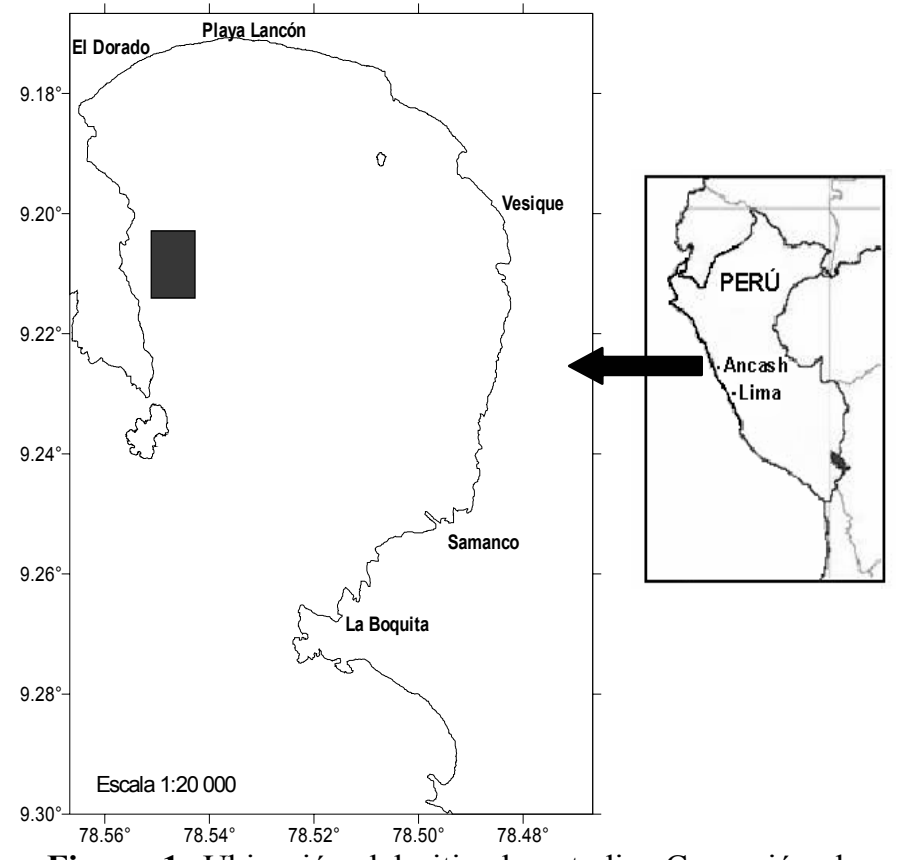

Figura 1. Ubicación del sitio de estudio, Concesión de Maricultura de la Universidad Ricardo Palma en la Bahía Samanco, Ancash, Perú.

\section{Materiales y Métodos}

El presente estudio se realizó en la Concesión Marina para la Maricultura de la Universidad Ricardo Palma, localizada en bahía Samanco ( $9^{\circ} 12^{\prime} \mathrm{S}, 78^{\circ}$ 30 'W), Ancash, Perú (Figura 1). El día 20 Octubre de 1998 se tomó una estructura de cultivo aleatoriamente que consistió en; una boya de reflote y señalizadora, una linterna, un pearl net, un cabo, proveniente de un long line que fue retirado para su limpieza. Se raspó con una espátula de metal cada uno de los materiales, teniendo cuidado en no dañar los organismos. Además se colectaron 10 ejemplares de concha de abanico los cuales presentaron bioincrustantes por encima de las valvas. Las especies colectadas, se fijaron en formalina al $10 \%$ en agua de mar para su posterior identificación en laboratorio. En este trabajo solo se consideró las especies de fauna.

\section{Resultados y discusión}

Se identificaron un total de 33 especies de bioincrustantes asociada a las diferentes estructuras de cultivo (Tabla 1). Las especies presentes correspondieron a 10 grupos taxonómicos: Crustacea (7), Bivalva (6), Gasteropoda (5), Polyplacophora (2), Brachiopoda (1), Echinodermata (4), Polychaeta (4), Hidroozoa (1), Hemichordata(1), Peces (2). El taxón mejor representado fue Crustacea (7 especies), seguida de Bivalva (6 especies) y Gasteropoda (5 especies). La estructura que tuvo mayor cantidad de bioincrustantes fue la boya (19 especies), seguida de la linterna (15 especies) y cuerdas (8 especies). Los bioincrustantes considerados como epibiontes estuvieron representado por 8 especies sobre cirripedos, 3 especies sobre las valvas de $A$. purpuratus y 1 especie sobre Tubularia sp. De las 33 especies el $45.4 \%(\mathrm{~N}=15)$ fueron suspensívoros, $15.1 \% \quad(\mathrm{~N}=5)$ carnívoras, $27.2 \%$ $(\mathrm{N}=9)$ herbívoras, $9.09 \%(\mathrm{~N}=3)$ omnívoras y $3.03 \%$ $(\mathrm{N}=1)$ depositívoras.

Las especies de invertebrados que conforman los bioincrustantes, son propias de hábitats intermareales y submareales (Paredes et al., 1988; Paredes et al., 1999; Romero et al., 1988) que aprovechan la disponibilidad de estas estructuras para colonizarlas. Sin embargo Planes minutus es un cangrejo pelágico que usualmente puede asentarse sobre ballenas, tortugas, o algas que flotan en superficie (Johnson \& Snook, 1955). Esta especie eventualmente aprovechó las estructuras como hábitat.

Este reporte de 33 especies, es número variable, ya que los muestreos se realizaron en los momentos en que las estructuras fueron cambiadas para su limpieza, por lo que la disponibilidad de estos sustratos varía en el tiempo de acuerdo al período en que permanece la estructura en contacto con el agua. En otros lugares de Latinoamérica se han reportado especies de bioincrustantes que se presentan también variables en número. Considerando solamente la fauna bioincrustantes tenemos; en bahía Guanaqueros (Chile) 40 especies, Golfo de Cariaco (Venezuela) 12 especies, en la ensenada de Pinheira (Brasil) 16 especies, Bahía Magdalena y Bahía de la Paz (Mexico) 38 especies (Uribe et al., 2001). Los Phyla registrados en los diferentes países son similares a los de este reporte, a excepción de las dos especies de peces registradas en este estudio. Otra fuente de variación en el número de especies puede atribuirse a que algunos individuos se desprenden del sustrato al ser trasladado al lugar de limpieza, así como también a diferentes tipos de esfuerzo de muestreo.

En bolsas colectoras, pearl nets y linternas de cultivo se encontraron Tubularia sp. y Ciona intestinalis. Juveniles de A. purpuratus también fueron registrados asentados en Tubularia sp., tal como mencionan Aguilar \& Stotz (2000), esta especie serviría como lugar de asentamiento de esta especie. Las especies que correspondieron a los hábitats intermareales i.e. Austromegabalanus psittacus, Balanus laevis, Pollicipes elegans, se encontraron principalmente en las boyas las cuales se presentan mas cerca de la superficie (las de reflote), y en la misma superficie, como las boyas señalizadoras.

Semimytilus algosus fue observado junto a Tubularia sp. La presencia de hidrozoos y mitilidos en las estructuras, también ha sido documentada en cultivo de Placopecten magellanicus en Baie des Chaleurs en Canadá, donde se reportan Tubularia larynx y Mytilus edulis (Claereboudt et al., 1994). Estas especies son de hábitats submareales, debido a que estas estructuras se ubican entre 8 y $20 \mathrm{~m}$ de profundidad en esta zona. 
Tabla 1. Principales especies de bioincrustantes indicando la estructura en que fueron encontradas $\mathrm{y}$ hábito trófico. $\mathrm{B}=\mathrm{Boya}, \mathrm{L}=$ Linterna, $\mathrm{C}=$ Cuerda, $\mathrm{PN}=$ Pearl net, $\mathrm{BC}=$ Bolsa Colectora, $\mathrm{E} 1=$ Epibionte sobre $A$. purpuratus, E2= Entre y sobre cirripedos, E3= Epibionte sobre Tubularia sp.

\begin{tabular}{|c|c|c|}
\hline Especie & Estructura & $\begin{array}{l}\text { Hábito } \\
\text { trófico }\end{array}$ \\
\hline \multicolumn{3}{|l|}{ Crustacea } \\
\hline $\begin{array}{l}\text { Austromegabalanus } \\
\text { psittacus }\end{array}$ & $\mathrm{B}, \mathrm{L}, \mathrm{C}$ & Suspensívoro \\
\hline Balanus laevis & B,L,E1 & Suspensívoro \\
\hline Pollicipes elegans & $\mathrm{L}, \mathrm{C}$ & Suspensívoro \\
\hline Lepas sp. & $\mathrm{B}, \mathrm{C}$ & Suspensívoro \\
\hline Pilumnoides perlatus & L,E2 & Carnívoro \\
\hline Pachicheles grossimanus & L,E2 & Carnívoro \\
\hline Planes minutus & $\mathrm{B}, \mathrm{E} 2$ & Carnívoro \\
\hline \multicolumn{3}{|l|}{ Bivalva } \\
\hline Argopecten purpuratus & $\mathrm{BC}, \mathrm{E} 3$ & Suspensívoro \\
\hline Semimytilus algosus & $\mathrm{C}, \mathrm{PN}, \mathrm{L}, \mathrm{E} 1, \mathrm{E} 2$ & Suspensívoro \\
\hline Lithophaga peruviana & $\mathrm{C}$ & Suspensívoro \\
\hline Barbatia gradata & $\mathrm{L}$ & Suspensívoro \\
\hline Pteria sterna & $\mathrm{L}, \mathrm{C}$ & Suspensívoro \\
\hline Hiatela solida & $\mathrm{BC}$ & Suspensívoro \\
\hline Crucibulum spinosum & B & Herbívoro \\
\hline Stramonita haemastoma & B & Omnívoro \\
\hline Stramonita biseralis & B & Omnívoro \\
\hline Scurria viridula & $\mathrm{B}, \mathrm{E} 2$ & Herbívoro \\
\hline Fissurella limbata & $\mathrm{B}, \mathrm{C}$ & Herbívoro \\
\hline \multicolumn{3}{|l|}{ Polyplacophora } \\
\hline Acanthopleura echinata & $\mathrm{B}, \mathrm{E} 2$ & Herbívoro \\
\hline Acanthopleura niger & $\mathrm{B}, \mathrm{E} 2$ & Herbívoro \\
\hline \multicolumn{3}{|l|}{ Brachiopoda } \\
\hline Discinisca lamellosa & E1,L,B & Suspensívoro \\
\hline \multicolumn{3}{|l|}{ Echinodermata } \\
\hline Tetrapygus niger & $\mathrm{L}, \mathrm{B}$ & Herbívoro \\
\hline Arbacia spatuligera & L,B & Herbívoro \\
\hline Caenocentrotus gibosus & L,B & Herbívoro \\
\hline Cucumaria dubiosa & $\mathrm{B}$ & Suspensívoro \\
\hline \multicolumn{3}{|l|}{ Polychaeta } \\
\hline Hydroides sp. & E2 & Suspensívoro \\
\hline Pomatoceros triqueter & E2 & Suspensívoro \\
\hline Nereis callaona & E2 & Carnívoro \\
\hline Lepidonotus sp. & E2 & Depositívoro \\
\hline \multicolumn{3}{|l|}{ Hidrozoa } \\
\hline \multicolumn{3}{|l|}{ Hemichordata } \\
\hline Ciona intestinalis & $\mathrm{BC}, \mathrm{L}, \mathrm{C}, \mathrm{PN}, \mathrm{B}$ & Suspensívoro \\
\hline Peces & $\mathrm{B}, \mathrm{E} 2$ & Omnívoro \\
\hline Hypsoblennius sordidus & & \\
\hline $\begin{array}{l}\text { Pseudobalistes } \\
\text { naufragium }\end{array}$ & $\mathrm{L}$ & Herbívoro \\
\hline
\end{tabular}

También encontramos conchas horadadoras como Hiatella solida y Litophaga peruviana, habitando entre los nudos de las cuerdas. En la linterna se identificaron individuos de Pteria sterna y Barbatia gradata, estas especies según Alamo \& Valdivieso (1999), se distribuyen desde Baja California hasta
Pimentel y desde Baja California hasta Paita en Perú respectivamente. La presencia de ambos bivalvos en la zona de estudio, por debajo del limite sur de su distribución, es atribuible al efecto producido por las corrientes cálidas asociadas al evento el Niño, que se distribuyeron a lo largo de la costa durante 1997 y 1998 (Paredes \& Cardoso, 1999).

El poliqueto tubícola Hidroides elegans y el braquiopodo Discinisca lamellosa se encontraron asentados en las valvas de A. purpuratus, cubriendo ambas valvas en su totalidad. Aunque nuestras observaciones no permiten decir que tipo de interacción hay entre estas especies y A. purpuratus, otros autores indican que los poliquetos (por ejemplo; Polydora sp.) han sido causantes de importantes mortalidades de A. purpuratus en Bahía Tongoy, Chile (Uribe et al., 2001).

Las observaciones muestran que las estructuras de cultivo suspendido de A. purpuratus, ofrecen sustratos para la colonización de bioincrustantes, las que llegan a desarrollar toda una comunidad. La mayoría de estas especies son filtradoras, lo que puede significar competencia para $A$. purpuratus y afectar su crecimiento. Otras especies pueden afectar el cultivo porque son depredadores de las conchas, como los cangrejos carnívoros y otros grupos de especies afectan el material porque lo deterioran e incrementan el peso de los sistemas. Estudios a futuro deberán enfocarse en medir de manera experimental el efecto de los bioincrustantes en la producción de $A$. purpuratus.

\section{Agradecimientos}

Los autores desean agradecer al Dr. V. Alamo (Q.E.P.D.) por su colaboración durante la realización del presente trabajo. A P. Huamán por su ayuda en la identificación de especies y a J. Riascos por sus comentarios a la presente nota. Dos revisores anónimos contribuyeron a la mejora del manuscrito final.

\section{Literatura citada}

Avendaño M., Cantillanez M., Le Pennec M., Lodeiros C. \& Freites L. 2001. Cultivo de pectínidos de Iberoamericanos en suspensión. En: A.N. MaedaMartínez (Ed.): Los moluscos pectínidos de Iberoamérica: Ciencia y Acuicultura. Cap.10: 193-211.

Alamo V. \& Valdivieso V. 1997. Lista Sistemática de Moluscos Marinos del Perú. 2da. Edición, Publicación Especial. Inst. Mar Callao-Perú.

Aguilar S. \& Mendo J. 2002. Análisis de la comunidad asociada a bolsas colectoras de concha de abanico Argopecten purpuratus en la Bahía Independencia. En: Mendo, J. \& M. Wolff. (Eds.). Memorias I Jornada Científica. Bases Ecológicas y Socioeconómicas para el Manejo de los Recursos Vivos de la Reserva Nacional de Paracas. 33-36 pp.

Aguilar M. \& Stotz W. 2000. Settlement sites of juveniles of scallop Argopecten purpuratus (Lamarck 1819) in the 
subtidal zone at Puerto Aldea, Tongoy Bay, Chile. J. Shellfish Res. 19: 749-755.

Claereboudt M.R., Bureau D., Cote \& Himmelman J.H. 1994. Bioincrustantes development and its effects on the growth of juvenile giant scallops (Placopecten magellanicus) in suspended culture. Aquaculture. 121: 327-342.

Johnson E. \& Snook H. 1955. Sea shore animals of the pacific coast. Dover Publications, INC. New York.

Lesser M.P., Shumway S., Cucci E. \& Smith J. 1992. Impact of bioincrustantes organisms on mussel rope culture: interespecific competition for food among suspensionfeeding invertebrates. J. Exp. Mar. Biol. Ecol. 165: 91102.

Paredes C., Tarazona J., Canahuire E., Romero L. \& Cornejo O. 1988. Invertebrados Macro-Bentónicos del área de Pisco, Perú. En: Salzwedel H. \& Landa A. (Eds.). Recursos y dinámica del ecosistema del afloramiento peruano. Bol. Inst. Mar Perú Callao Vol. Extraor.: 121-132.

Paredes C. \& Cardoso F. 1999. Fauna tropical y su relación con "El Niño". In: Libro de Resumenes. Seminario Taller: Impacto de los Eventos "El Niño Oscilación Sur" sobre la diversidad Biológica de América. Consejo Nacional de Ciencia y Tecnología. Lima - Perú. 1 - 4.

Paredes C., Cardoso F. \& Tarazona J. 1999. Invertebrados del intermareal rocoso del departamento de Lima, Perú: una lista comentada de especies. Rev. Per. Biol. 6(1): 143-151.

Perea M., Ballesteros M. \& Turo X. 1990. Estudio de los organismos epibióntes en un cultivo de bivalvos marinos en el delta del Ebro. Cahiers de Biologie Marine. 31: 385-399.

Romero L., Paredes C. \& Chavez R. 1988. Estructura de la macrofauna asociada a los rizoides de Lessonia sp. (Laminariales, Phaeophyta). En: Salzwedel H. \& Landa A. (Eds). Recursos y Dinámica del Ecosistema de Afloramiento Peruano. Bol. Inst. Mar Perú Callao. Vol. Extraor: $133-139$.

Ross K.A., Thorpe J.P., Norton T.A. \& Brand A.R. 2002. Bioincrustantes in scallop cultivation: help or hindrance? J. Shellfish Res. 21: 539-547.

Thouzeau G. 1991. Experimental collection of postlarvae of Pecten maximus (L.) and other benthic macrofaunal species in the Bay Saint-Brieuc, France. I. Settlement patterns and biotic interactions among the species collected. J. Exp. Mar. Biol. Ecol. 148: 159-179.

Uribe E., Lodeiros C., Felix-Pico E. \&Etchepare I. 2001. Epibiontes en pectínidos de Iberoamerica. En: MaedaMartínez A.N. (Ed.): Los moluscos pectínidos de Iberoamérica: Ciencia y Acuicultura. Cap.13: 249-266.

Widman J. \& Rodhes E. 1991. Nursery culture of the bay scallop, Argopecten irradians irradians, in suspended mesh nets. Aquaculture. 99: 257-267.

1. Instituto de Investigaciones Oceanológicas, Universidad de Antofagasta. Av. Angamos 601. Antofagasta, Chile. Telefono: 56(55)637-404, Email: apacheco@uantof.cl

2. Rainforest Expeditions. Av. Aramburu 166, Dpto. 4B, Lima 18. Email: balanusperu@yahoo.com.mx 